\title{
Effective theory and universal relations for Fermi gases near a $d$-wave-interaction resonance
}

\author{
Pengfei Zhang, ${ }^{1}$ Shizhong Zhang, ${ }^{2, *}$ and Zhenhua $\mathrm{Yu}^{3, \dagger}$ \\ ${ }^{1}$ Institute for Advanced Study, Tsinghua University, Beijing 100084, China \\ ${ }^{2}$ Department of Physics and Center of Theoretical and Computational Physics, The University of Hong Kong, Hong Kong, China \\ ${ }^{3}$ School of Physics and Astronomy and TianQin Research Center for Gravitational Physics, Sun Yat-Sen University, Zhuhai 519082, China
}

(Received 24 May 2016; revised manuscript received 11 November 2016; published 10 April 2017)

\begin{abstract}
In this work, we present an effective-field theory to describe a two-component Fermi gas near a $d$-waveinteraction resonance. The effective-field theory is renormalizable by matching with the low-energy $d$-wave scattering phase shift. Based on the effective-field theory, we derive universal properties of the Fermi gas by the operator-product-expansion method. We find that beyond the contacts defined by adiabatic theorems, the asymptotic expressions of the momentum distribution and the Raman spectroscopy involve two extra contacts which provide additional information on correlations of the system. Our formalism sets the stage for further explorations of many-body effects in a $d$-wave resonant Fermi gas. Finally, we generalize our effective-field theory for interaction resonances of arbitrary higher partial waves.
\end{abstract}

DOI: 10.1103/PhysRevA.95.043609

\section{INTRODUCTION}

Correlations of $d$-wave symmetry are of fundamental interest in modern physics. One outstanding example is the $d$-wave Cooper pairing in high- $T_{c}$ superconductors, which provides a paradigmatic case of strongly correlated electron systems [1]. In cold-atom systems, strong $d$-wave correlations can also be generated close to a $d$-wave Feshbach resonance, as has been demonstrated experimentally in $\mathrm{Cr}[2,3]$. While it is generally believed that, compared with $s$-wave resonance, atomic gases close to higher partial wave resonances suffer more rapid atom loss, recent spectroscopic measurements around a $p$-wave Feshbach resonance indicate that quasiequilibrium states of such systems exist and their universal properties can be investigated [4]. Theoretically, however, many-body physics with resonant $d$-wave interactions has been rarely studied, and in particular, an appropriate minimal model is still lacking.

In this work, we consider a two-component Fermi gas near a $d$-wave-interaction resonance. We construct an effective low-energy field theory, the bare coupling constants of which are renormalized by matching with the $d$-wave scattering phase shift $\cot \delta(k)=-1 /\left(D k^{5}\right)-1 /\left(v k^{3}\right)-1 /(R k)$. The supervolume $D$, the effective volume $v$, and the effective range $R$ are the minimal set of parameters that is needed to parametrize the interfermion interactions. Furthermore, we use the effective theory, combined with the operator-productexpansion (OPE) method, to derive universal properties of the Fermi gas when the average interparticle distance is much larger than the range $r_{0}$ associated with the interfermion interaction. We find that the universal behavior of the system is governed by five quantities, three of which are related to the variation of the system energy with respect to the three $d$-wave scattering parameters, analogous to the contacts defined in the case of the $s$ - and $p$-wave cases [5-15]. However, we find that the subleading terms of the tails of momentum distribution and Raman spectroscopy involve two additional contacts, which further characterize the correlations of the system at

\footnotetext{
*shizhong@hku.hk

†huazhenyu2000@gmail.com
}

short distances. Our effective-field theory provides a minimal model for studying other many-body physics of Fermi gases near a $d$-wave resonance. We show that the $d$-wave contacts reveal much richer correlation structures than the $s$-wave case. Finally, we generalize our formalism for resonant interactions to arbitrary higher partial waves.

\section{EFFECTIVE-FIELD THEORY}

To describe the low-energy degrees of freedom close to a $d$-wave-interaction resonance, we adopt a Lagrangian field theory and require that the Lagrangian density obey the following symmetry requirements: (1) rotation symmetry and (2) Galilean invariance such that the scattering of two fermions in vacuum does not depend on their center-of-mass momentum. In addition, we aim to establish a local effectivefield theory, which should be renormalizable in the low-energy limit in terms of the minimal set of scattering parameters $D, v, R$, describing the $d$-wave scattering phase shift.

The Lagrangian density of the effective-field theory that we construct for the system up to a momentum cutoff $\Lambda$ is given by

$$
\begin{aligned}
\mathcal{L}= & \sum_{i=1}^{2} \psi_{i}^{\dagger}\left(i \partial_{t}+\frac{\nabla^{2}}{2 M}\right) \psi_{i}+\sum_{m=-\ell}^{\ell} \bar{g}\left(d_{\ell m}^{\dagger} \mathcal{Y}_{m}+\text { H.c. }\right) \\
& +\eta \sum_{m=-\ell}^{\ell} d_{\ell m}^{\dagger}\left[i \partial_{t}+\frac{\nabla^{2}}{4 M}+\bar{z}\left(i \partial_{t}+\frac{\nabla^{2}}{4 M}\right)^{2}-\bar{v}\right] d_{\ell m},
\end{aligned}
$$

where $\ell=2$ and the operator $\mathcal{Y}_{m}$ is given by

$$
\begin{aligned}
\mathcal{Y}_{m}= & \frac{1}{4} \sum_{a, b=x, y, z} C_{a b}^{m}\left[\left(\partial_{a} \psi_{1}\right)\left(\partial_{b} \psi_{2}\right)-\left(\partial_{a} \partial_{b} \psi_{1}\right) \psi_{2}\right. \\
& \left.+\left(\partial_{b} \psi_{1}\right)\left(\partial_{a} \psi_{2}\right)-\psi_{1}\left(\partial_{a} \partial_{b} \psi_{2}\right)\right] .
\end{aligned}
$$

The field operator $\psi_{i}$ is the annihilation operator for fermions in state $|i\rangle . M$ is the mass of the fermions. We take $\hbar=1$ throughout. The dimer fields $d_{\ell m}$ of azimuthal quantum number $m$ mediate the $d$-wave interaction between the two fermions, which we assume to be isotropic. $C_{a b}^{m}$ are the Clebsch-Gordan coefficients when transforming $k_{i} k_{j} / k^{2}$ to 
the spherical harmonics $\sqrt{4 \pi} Y_{2 m}(\hat{k})$. In terms of $a_{i, \mathbf{k}}$ and $b_{\ell m, \mathbf{k}}$, the Fourier transformations of the operators $\psi_{i}$ and $d_{\ell m}$, the fermion-dimer coupling in the Lagrangian $L=\int d \mathbf{r} \mathcal{L}$ [the second term in Eq. (1)] takes the form

$$
L_{f d}=\bar{g} \sqrt{\frac{4 \pi}{V}} \sum_{m=-\ell}^{\ell} \sum_{\mathbf{p}, \mathbf{k}}\left[k^{\ell} Y_{\ell m}(\hat{k}) b_{\ell m, \mathbf{p}}^{\dagger} a_{1, \frac{\mathbf{p}}{2}+\mathbf{k}} a_{2, \frac{\mathbf{p}}{2}-\mathbf{k}}+\text { H.c. }\right],
$$

where $V$ is the volume of the system. Since we focus on the effects of the $d$-wave resonance, we neglect possible background scatterings of either $s$ - or $p$-wave symmetry and those due to direct couplings between the fermions. The term proportional to $\eta= \pm 1$ describes the energy of a single dimer, with $\bar{v}$ being its detuning. Unlike the case for $p$-wave scattering, an extra term proportional to the bare coupling constant $\bar{z}$ is constructed in order to renormalize the effective range $R$ [see Eq. (9)] while still respecting the Galilean invariance. As will be shown later, it is necessary to take $\eta=-1$ in order to achieve a renormalizable theory.

The effective-field theory in Eq. (1) differs from that for the $s$-wave and $p$-wave resonance models, and it is worthwhile to point out the differences. In the $s$-wave case, Kaplan was the first to use an $s$-wave dimer field $b_{00, \mathbf{k}}$ to describe the nonrelativistic scattering between nucleons with a large $s$-wave $(\ell=0)$ scattering length $a_{s}$ [16]. In this case, the zerorange limit $\Lambda \rightarrow \infty$ is well defined with the choice $\eta=1$ and $\bar{z}=0$ by matching the scattering matrix with the $s$-wave phase-shift expansion $k \cot \delta_{s}(k)=-1 / a_{s}$. The same resonance model was constructed independently by Kokkelmans et al. for atoms close to an $s$-wave Feshbach resonance [17], for which the dimer field $b_{00, \mathbf{k}}$ naturally represents the closed-channel molecules.

Different from the $s$-wave case, low-energy scattering in the $p$-wave channel is described by two parameters, $k^{3} \cot \delta_{p}(k)=-1 / v_{p}-k^{2} / R_{p}$ [18]. Here $v_{p}$ is the $p$-wave scattering volume, and $R_{p}$ is the $p$-wave effective range. In this case, however, to obtain a renormalizable theory with finite $v_{p}$ and $R_{p}$ in the low-energy limit, one has to take $\eta=-1$. This means that the free dimer field $b_{1 m, \mathbf{k}}$ becomes a ghost field with a negative norm [18]. However, such a negative norm is relevant only at a much higher energy, of the order of $\Lambda^{2}$, which is irrelevant for the low-energy physics described by the scattering phase shift $\delta_{p}(k)$.

In the $d$-wave-interaction resonance, it is first important to note that the low-energy scattering phase shift must be retained up to order $k^{4}$, namely, $k^{5} \cot \delta_{d}(k)=-1 / D-k^{2} / v-k^{4} / R$; the three interaction parameters $D, v$, and $R$ are the minimal set. This is because across the resonance, while the magnitude of $D$ can be tuned to be much larger than the interaction range $r_{0}, v / r_{0}^{3}$ and $R / r_{0}$ are typically of order unity. Taking the zero limit $v \rightarrow 0$ or (and) $R \rightarrow 0$ would lead to the noninteracting limit, i.e., $\delta(k) \rightarrow 0$, which cannot describe the original interacting system. In contrast, it is safe to take the zero limit of the expansion coefficients of order higher than $k^{4}$ in $k^{5} \cot \delta(k)$. Now, we note that in Eq. (1), the term $d_{\ell m}^{\dagger}\left(i \partial_{t}\right) d_{\ell m}$ corresponds to the total energy of two scattering fermions, and the term $d_{\ell m}^{\dagger}\left(-\nabla^{2} / 4 M\right) d_{\ell m}$ corresponds to the centerof-mass energy. The combination $d_{\ell m}^{\dagger}\left(i \partial_{t}+\nabla^{2} / 4 M\right) d_{\ell m}$ thus

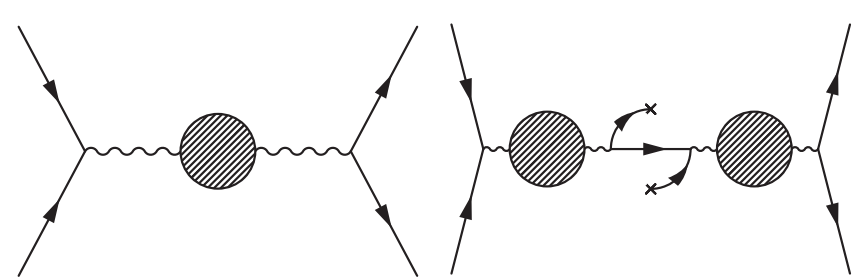

(a)

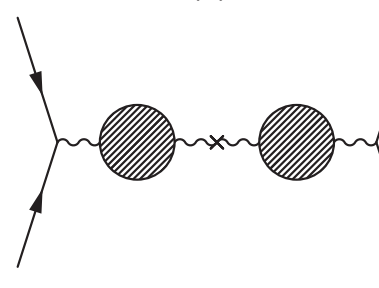

(b)

(c)

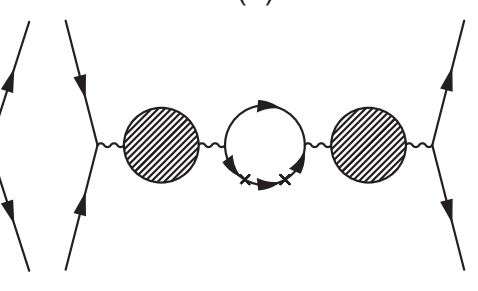

(d)

FIG. 1. Feynman diagrams for (a) the $T$ matrix for two fermions, (b) the matrix element of $\psi_{i}^{\dagger}(\mathbf{R}+\mathbf{r} / 2) \psi_{i}(\mathbf{R}-\mathbf{r} / 2)$, (c) the matrix element of dimer bilinears, and (d) the diagram for the Raman spectrum. In these diagrams, the wavy lines represent the propagators for the bare dimer fields, the solid lines represent the propagators for the bare fermion fields, and the crosses represent the operators which are inserted.

corresponds to the relative scattering energy. As a result, we explicitly construct the extra term $\bar{z} d_{\ell m}^{\dagger}\left(i \partial_{t}+\nabla^{2} / 4 M\right)^{2} d_{\ell m}$ in Eq. (1) to match the $k^{4}$ dependence of $k^{5} \cot \delta_{d}(k)$ for $d$-wave resonances. Note that by construction, the Lagrangian equation (1) maintains explicitly the Galilean invariance.

The renormalizability of Eq. (1) is manifested by calculating the $T$ matrix, $T\left(\mathbf{P}, \mathbf{k}, \mathbf{k}^{\prime}, \Omega\right)$, of scattering between two fermions with relative incoming (outgoing) momentum $2 \mathbf{k}$ $\left(2 \mathbf{k}^{\prime}\right)$ and total momentum $\mathbf{P}$. Due to the Galilean invariance of Eq. (1), one needs to calculate only in the center-of-mass frame, and the $T$ matrix is given by

$$
T_{m}\left(\mathbf{0}, \mathbf{k}, \mathbf{k}^{\prime}, \Omega\right)=-4 \pi \bar{g}^{2} k^{4} Y_{2 m}(\hat{\mathbf{k}}) Y_{2 m}^{*}\left(\hat{\mathbf{k}}^{\prime}\right) \mathcal{D}(\mathbf{0}, \Omega),
$$

where $|\mathbf{k}|=\left|\mathbf{k}^{\prime}\right|$ due to energy conservation and $\hat{\mathbf{k}}=\mathbf{k} /|\mathbf{k}|$ and $\hat{\mathbf{k}}^{\prime}=\mathbf{k}^{\prime} /\left|\mathbf{k}^{\prime}\right| . \mathcal{D}(\mathbf{P}, \Omega)$ is the full dimer propagator, given in Fig. 1(a),

$$
\begin{aligned}
& \mathcal{D}^{-1}(\mathbf{P}, \Omega) \\
& \quad=\overline{\mathcal{D}}^{-1}(\mathbf{P}, \Omega)-\frac{\bar{g}^{2}}{2 \pi^{2}} \int_{0}^{\Lambda} d q \frac{q^{6}}{\Omega-P^{2} / 4 M-q^{2} / M},
\end{aligned}
$$

where $\overline{\mathcal{D}}(\mathbf{P}, \Omega)$ is the bare dimer propagator given by

$$
\overline{\mathcal{D}}(P, \Omega)=\frac{E_{p,+}-E_{p,-}}{\eta \bar{z}}\left(\frac{1}{\Omega-E_{p,+}}-\frac{1}{\Omega-E_{p,-}}\right),
$$

with $E_{p, \pm}=P^{2} / 4 M-(1 \mp \sqrt{1+4 \bar{v} \bar{z}}) / 2 \bar{z}$ being the dimers' normal-mode energies. In the case $1+4 \bar{v} \bar{z}>0$, there always exists one branch of $\overline{\mathcal{D}}(P, \Omega)$ with negative weight corresponding to the presence of ghost fields [19], irrespective of the sign of $\eta$. The appearance of ghost fields is inevitable due to the requirement to renormalize not only $v$ but also $R$ for $d$-wave interactions [see Eqs. (8) and (9)] [18]. In the case $1+4 \bar{\nu} \bar{z}<0$, the poles of $\overline{\mathcal{D}}(P, \Omega)$ move away from the 
TABLE I. Differences between our $d$-wave effective-field theory with the $s$ - and $p$-wave cases. Each case is renormalized to the minimal interaction parameters listed.

\begin{tabular}{lccccc}
\hline \hline & $\ell$ & Minimal parameters & $\eta$ & $\bar{z}$ & Ghost field \\
\hline$s$-wave & 0 & $a_{s}$ & 1 & 0 & No \\
$p$-wave & 1 & $v_{p}, R_{p}$ & -1 & 0 & Yes \\
$d$-wave & 2 & $D, v, R$ & -1 & $\neq 0$ & Yes \\
\hline \hline
\end{tabular}

real axis into the complex plane and $\bar{D}(P, \Omega)$ by itself seems problematic. However, the low-energy observables predicted by the full coupled effective-field theory remain valid (see below). In Table I, we summarize the main differences between our $d$-wave effective-field theory with the $s$ - and $p$-wave cases.

Matching $T_{m}\left(\mathbf{0}, k \hat{\mathbf{k}}, k \hat{\mathbf{k}}^{\prime}, k^{2} / M+i 0\right) \quad$ with $\cot \delta_{d}(k)=$ $-1 / D k^{5}-1 / v k^{3}-1 / R k$ in the limit $k \rightarrow 0$, we find the renormalization conditions:

$$
\begin{aligned}
\frac{1}{D} & =-\eta \frac{4 \pi \bar{v}}{\bar{g}^{2} M}+\frac{2 \Lambda^{5}}{5 \pi}, \\
\frac{1}{v} & =\eta \frac{4 \pi}{\bar{g}^{2} M^{2}}+\frac{2 \Lambda^{3}}{3 \pi}, \\
\frac{1}{R} & =\eta \frac{4 \pi \bar{z}}{\bar{g}^{2} M^{3}}+\frac{2 \Lambda}{\pi} .
\end{aligned}
$$

To keep values of $D, v$, and $R$ finite while taking the limit $\Lambda \rightarrow$ $\infty$, we require $\eta=-1$. Otherwise, if $\eta=1$, from Eq. (8), $|v|<3 \pi / 2 \Lambda^{3}$ and approaches zero. In fact, it turns out not to be possible to construct a purely fermionic model with contact interfermion interactions which reproduces the correct $d$-wave low-energy scattering amplitude with finite parameters $v$ and $R$ in the limit $\Lambda \rightarrow \infty$. Thus it is crucial to introduce the dimer field with the concomitant appearance of the ghost field, which, however, does not alter the low-energy physics.

The applicable regime of our effective-field theory can be analyzed from the pole structure of $T_{m}$ in terms of the renormalized parameters,

$$
\begin{aligned}
& T_{m}\left(\mathbf{0}, k \hat{\mathbf{k}}, k \hat{\mathbf{k}}^{\prime}, \Omega\right) \\
& \quad=-\frac{16 \pi^{2} k^{4} Y_{2 m}(\hat{\mathbf{k}}) Y_{2 m}^{*}\left(\hat{\mathbf{k}}^{\prime}\right) / M}{1 / D+M \Omega / v+(M \Omega)^{2} / R+i(M \Omega)^{5 / 2}} .
\end{aligned}
$$

For simplicity, let us consider the limit $1 / D \rightarrow 0^{+}$. The real pole of $T_{m}$ at $\Omega \rightarrow 0^{-}$with positive weight $\sim v$ corresponds to a physical two-fermion bound state approaching threshold. However, since, typically, $v \sim r_{0}^{3}$ and $R \sim r_{0}$, there are other complex poles at energies $|\Omega| \sim 1 / M r_{0}^{2}$, which apparently violate the unitary condition of the $S$ matrix. The origin of these unphysical poles is the truncation of $\cot \delta_{d}(k)$. However, as long as we are interested only in energy scales much smaller than $1 / M r_{0}^{2}$, which will be the case in the following, our effective-field theory should give physically valid results.

The negative norm ghost dimer fields are introduced as a convenient way to formulate a local field theory. If one instead uses a closed-channel Feshbach dimer state whose internal wave function is $\phi(r) Y_{\ell m}(\hat{\mathbf{r}})$, with $\mathbf{r}$ being the relative coordinates between two constituent fermions, the free dimer part of the Lagrangian is normal, i.e., $\sum_{\mathbf{k}} b_{\ell m, \mathbf{k}}^{\dagger}\left(i \partial_{t}-\right.$ $\left.k^{2} / 4 M\right) b_{\ell m, \mathbf{k}}$, while the fermion-dimer coupling part becomes $(1 / \sqrt{V}) \sum_{\mathbf{P}, \mathbf{k}}\left[\tilde{\phi}(k) Y_{\ell m}(\hat{\mathbf{k}}) b_{\ell m, \mathbf{P}}^{\dagger} a_{1, \mathbf{P} / 2+\mathbf{k}} a_{2, \mathbf{P} / 2-\mathbf{k}}+\right.$ H.c. $]$, with $\tilde{\phi}(k)=\int_{0}^{\infty} d r r^{2} \phi(r) j_{\ell}(k r)$. It would render the theory nonlocal if one assumes any model form for $\tilde{\phi}(k)$, which would make it impossible to apply techniques for local effective-field theory, such as the operator product expansion discussed below. Of course, at this stage one can retrieve a seemingly local theory by asymptotically expanding $\tilde{\phi}(k)=k^{\ell} \sum_{n} \beta_{n} k^{2 n}$ and truncating the expansion at a certain order of $k$; in the $d$-wave case, the finite number of $\beta_{n}$ retained shall be renormalized to $D, v$, and $R$. However, by this approach, one can show that it is impossible to keep $D, v$, and $R$ finite simultaneously in the limit $\Lambda \rightarrow \infty$. The above distinction motivates us to employ the ghost dimer fields rather than the Feshbach dimers in our formalism.

\section{III. $d$-WAVE CONTACTS}

Effective-field theory has served as an ideal formalism to elucidate the universal aspects of quantum gases [20,21], in particular, the derivation of universal relations involving the so-called contacts using the OPE $[6,9,19,21-28]$. This is an operator relation for the product of two operators at small separation $[22,29]$

$$
O_{i}\left(\mathbf{R}+\frac{\mathbf{r}}{2}\right) O_{j}\left(\mathbf{R}-\frac{\mathbf{r}}{2}\right)=\sum_{l} f_{l}^{i j}(\mathbf{r}) O_{l}(\mathbf{R}),
$$

where $O_{i}$ are the local operators and $f_{l}^{i j}(\mathbf{r})$ are the expansion functions. A similar expansion can also be carried out in the time domain. OPE is an ideal tool to explore short-range physics, $r_{0} \ll r \ll n^{-1 / 3}$ in a field-theory context. Here $n$ is the average density.

In the case of $d$-wave interactions, we first define three contact densities (operators) as the derivatives of the Lagrangian density $\mathcal{L}$ with respect to $D^{-1}, v^{-1}$, and $R^{-1}$ by using Eqs. (7) to (9):

$$
\begin{gathered}
\frac{\hat{\mathcal{C}}_{D}}{M} \equiv \frac{\delta \mathcal{L}}{\delta\left(D^{-1}\right)}=\frac{M \bar{g}^{2}}{4 \pi} \sum_{m} d_{\ell m}^{\dagger} d_{\ell m}, \\
\frac{\hat{\mathcal{C}_{v}}}{M} \equiv \frac{\delta \mathcal{L}}{\delta\left(v^{-1}\right)}=\frac{M^{2} \bar{g}^{2}}{4 \pi} \sum_{m} d_{\ell m}^{\dagger}\left(i \partial_{t}+\frac{\nabla^{2}}{4 M}\right) d_{\ell m}, \\
\frac{\hat{\mathcal{C}}_{R}}{M} \equiv \frac{\delta \mathcal{L}}{\delta\left(R^{-1}\right)}=\frac{M^{3} \bar{g}^{2}}{4 \pi} \sum_{m} d_{\ell m}^{\dagger}\left(i \partial_{t}+\frac{\nabla^{2}}{4 M}\right)^{2} d_{\ell m} .
\end{gathered}
$$

Note that we have used the equation of motion satisfied by $d_{\ell m}$ to obtain the concise expression of Eq. (13). While $\hat{\mathcal{C}}_{D}$ is proportional to the total dimer density, $\hat{\mathcal{C}}_{v}$ and $\hat{\mathcal{C}}_{R}$ can be considered proportional to the ones weighted by the powers of the internal energy of the dimers. A similar structure has been found for $p$-wave contacts [13]. In addition, as we will see from the tails of the momentum distribution and the Raman spectroscopy, it is also useful to introduce two extra $d$-wave contact densities as

$$
\begin{gathered}
\frac{\hat{\mathcal{C}}_{D, P}}{M} \equiv \frac{M^{2} \bar{g}^{2}}{4 \pi} \sum_{m} d_{\ell m}^{\dagger}\left(-\frac{\nabla^{2}}{4 M}\right) d_{\ell m}, \\
\frac{\hat{\mathcal{C}}_{v, P}}{M} \equiv \frac{M^{3} \bar{g}^{2}}{4 \pi} \sum_{m} d_{\ell m}^{\dagger}\left(i \partial_{t}+\frac{\nabla^{2}}{4 M}\right)\left(-\frac{\nabla^{2}}{4 M}\right) d_{\ell m},
\end{gathered}
$$


which, compared with Eqs. (12) and (13), are further weighted by the kinetic energy of the dimers and encapsulate additional information of correlations at short distances. The spatial integration of the expectation values of the contact densities are defined as the $d$-wave contacts: $C_{D}=\int d \mathbf{r}\left\langle\hat{\mathcal{C}}_{D}\right\rangle$, $C_{v}=\int d \mathbf{r}\left\langle\hat{\mathcal{C}}_{v}\right\rangle, \quad C_{R}=\int d \mathbf{r}\left\langle\hat{\mathcal{C}}_{R}\right\rangle, \quad C_{D, P}=\int d \mathbf{r}\left\langle\hat{\mathcal{C}}_{D, P}\right\rangle$, and $C_{v, P}=\int d \mathbf{r}\left\langle\hat{\mathcal{C}}_{v, P}\right\rangle$. From Eqs. (12)-(14), one can write down the adiabatic theorems,

$$
\frac{\partial F}{\partial \alpha^{-1}}=-\frac{C_{\alpha}}{M}, \quad \alpha=D, v, R,
$$

where $F$ is the free energy of the system. To illustrate the use of the effective-field theory, we now derive some universal relations between the introduced contacts and various physical observables.

\section{SHORT-DISTANCE EXPANSION}

The tails of the momentum distribution can be extracted from the one-body density matrix $\rho_{i}(\mathbf{R}, \mathbf{r})=\left\langle\psi_{i}^{\dagger}(\mathbf{R}+\right.$ $\left.\mathbf{r} / 2) \psi_{i}(\mathbf{R}-\mathbf{r} / 2)\right\rangle$ and can be measured experimentally by the time-of-flight technique [30,31]. To relate $\rho_{i}(\mathbf{R}, \mathbf{r})$ to the $d$-wave contacts, we calculate the OPE by matching the matrix elements of operators from an incoming state $|I\rangle$ with two fermions of different species having momenta $\mathbf{P} / 2+k \hat{\mathbf{k}}$ and $\mathbf{P} / 2-k \hat{\mathbf{k}}$ to an outgoing state $|F\rangle$ with two fermions having momenta $\mathbf{P} / 2+k \hat{\mathbf{k}}^{\prime}$ and $\mathbf{P} / 2-k \hat{\mathbf{k}}^{\prime}$. The total energy of the fermion pair is $E=P^{2} / 4 M+k^{2} / M$. Since we are interested in the rotationally invariant case, we will average over the direction of the total momentum $\mathbf{P}$. The case without rotational invariance can be calculated similarly. The matrix element of $\rho_{i}$ is given by the diagram shown in Fig. 1(b), and the result is

$$
\begin{aligned}
\left\langle F\left|\rho_{i}(\mathbf{R}, \mathbf{r})\right| I\right\rangle= & 4 \pi M^{2} \bar{g}^{4} k^{4} \sum_{m} Y_{2 m}(\hat{\mathbf{k}}) Y_{2 m}^{*}\left(\hat{\mathbf{k}}^{\prime}\right) \mathcal{D}^{2}(P, E) \\
& \times\left[\delta(\mathbf{r})+\frac{k^{2}}{2 \pi r}-\frac{3 r\left(k^{4}+P^{2} k^{2} / 18\right)}{8 \pi}\right] \\
& + \text { const }+o(\mathbf{r}) .
\end{aligned}
$$

Likewise, we calculate the matrix elements of the contact densities according to the diagrams shown in Figs. 1(a) and $1(\mathrm{~b})$. We find

$$
\begin{gathered}
\left\langle F\left|\hat{\mathcal{C}}_{D}\right| I\right\rangle=M^{2} \bar{g}^{4} k^{4} \sum_{m} Y_{2 m}(\hat{\mathbf{k}}) Y_{2 m}^{*}\left(\hat{\mathbf{k}}^{\prime}\right) \mathcal{D}^{2}(P, E), \\
\left\langle F\left|\hat{\mathcal{C}}_{v}\right| I\right\rangle=k^{2}\left\langle F\left|\hat{\mathcal{C}}_{D}\right| I\right\rangle \\
\left\langle F\left|\hat{\mathcal{C}}_{R}\right| I\right\rangle=k^{4}\left\langle F\left|\hat{\mathcal{C}}_{D}\right| I\right\rangle \\
\left\langle F\left|\hat{\mathcal{C}}_{D, P}\right| I\right\rangle=P^{2}\left\langle F\left|\hat{\mathcal{C}}_{D}\right| I\right\rangle / 4 \\
\left\langle F\left|\hat{\mathcal{C}}_{v, P}\right| I\right\rangle=P^{2} k^{2}\left\langle F\left|\hat{\mathcal{C}}_{D}\right| I\right\rangle / 4
\end{gathered}
$$

After Fourier transforming Eq. (18) and matching with Eqs. (19) to (23), we find that the momentum distribution $n_{i}(\mathbf{q})$ of the $i$ th species has a tail in the large- $q$ limit $\left(n^{1 / 3} \ll q \ll 1 / r_{0}\right)$,

$$
n_{i}(\mathbf{q})=\frac{1}{V}\left[\frac{C_{D}}{2 \pi^{2}}+\frac{C_{v}}{\pi^{2} q^{2}}+\frac{9 C_{R}+2 C_{v, P}}{6 \pi^{2} q^{4}}\right],
$$

whose magnitude depends on the $d$-wave contact densities. The presence of the additional quantity $C_{v, P}$, which cannot be derived from the adiabatic theorems (17), in the momentum tail can be understood in the following way. Let us consider a single pair of interacting fermions. In the center-of-mass frame of the pair where $C_{v, P}$ is zero according to Eqs. (16) and (24), the momentum tail $n_{\text {com }}(\mathbf{q})$ involves only $C_{\alpha}$ for $\alpha=D, v, R$. However, when we switch to a reference frame moving with a relative velocity $\mathbf{u}$, the momentum tail of the pair in this new frame should be $n(\mathbf{q})=n_{\text {com }}(\mathbf{q}-m \mathbf{u})$. Expansion of $n_{\text {com }}(\mathbf{q}-$ $m \mathbf{u}$ ) to order $1 / q^{4}$ leads to an extra term $\sim u^{2} C_{v}$ in $n(\mathbf{q})$, which is exactly the generally nonzero $C_{v, P}$ term in Eq. (24) in this case. Note that the Galilean invariance guarantees $C_{D}$ and $C_{v}$ have the same values in different reference frames [see Eq. (17)]. Quantities similar to $C_{v, P}$ have been introduced for $p$-wave interactions in three dimensions $[13,32,33]$.

The tails of the momentum distribution $n_{i}(\mathbf{q})$ seem to yield a divergent number of fermions. Actually, by the U(1) gauge invariance of Eq. (1), the conserved total particle number is given by

$$
\begin{aligned}
\hat{N}= & \int d \mathbf{r}\left(\sum_{i=1,2} \psi_{i}^{\dagger} \psi_{i}\right. \\
& \left.-\sum_{m}\left\{d_{m}^{\dagger}\left[1+\bar{z}\left(2 i \partial_{t}+\nabla^{2} / 2 M\right)\right] d_{m}+\text { H.c. }\right\}\right) .
\end{aligned}
$$

Using the renormalization relations (7), (8), and (9), one can verify that the divergent part of $n_{i}(\mathbf{q})$ at large $q$ is canceled by the dimer terms; the dimer terms can be considered counterterms to the fermion densities. Note that the factor $\bar{z}\left(2 i \partial_{t}+\nabla^{2} / 2 M\right)$ is due to the expansion of the bare dimer fields in terms of their normal modes.

\section{SHORT DISTANCE AND TIME EXPANSION}

The single-particle spectral function, which reveals fundamental properties of an interacting many-body system, such as pairing and pseudogap phenomena, can be measured using Raman spectroscopy in atomic gases [34,35]. When two Raman lasers of frequency $\omega_{1}$ and $\omega_{2}$ and wave vectors $\mathbf{k}_{1}$ and $\mathbf{k}_{2}$ are applied, atoms can be excited from the initial internal state $|2\rangle$ to the final internal state $|3\rangle$ by absorbing energy $\omega=\left|\omega_{1}-\omega_{2}\right|$ and momentum $\mathbf{q}=\mathbf{k}_{1}-\mathbf{k}_{2}$. The resultant number of atoms transferred to state $|3\rangle$ is, by the Fermi golden rule, proportional to the rate

$$
\begin{gathered}
I_{\mathrm{Ra}}(\mathbf{q}, \omega)=-\frac{1}{\pi} \operatorname{Im} \Pi_{\mathrm{Ra}}(\mathbf{q}, \omega) \\
\Pi_{\mathrm{Ra}}(\mathbf{q}, \omega)=-i V \int d t d \mathbf{r} e^{i \omega t-i \mathbf{q} \cdot \mathbf{r}}\left\langle T \mathcal{Q}_{23}(\mathbf{r}, t) \mathcal{Q}_{23}^{\dagger}(\mathbf{0}, 0)\right\rangle
\end{gathered}
$$

with $\mathcal{Q}_{23}(\mathbf{r}, t) \equiv \psi_{3}^{\dagger}(\mathbf{r}, t) \psi_{2}(\mathbf{r}, t)$. 
By calculating the OPE of $\mathcal{Q}_{23}(\mathbf{r}, t) \mathcal{Q}_{23}^{\dagger}(\mathbf{0}, 0)$ in both the time and space domains, we find for $\omega>\epsilon_{q} \equiv q^{2} / 2 M$

$$
\begin{aligned}
\frac{\pi}{M} I_{\mathrm{Ra}}(\mathbf{q}, \omega)= & \left(M \omega-\frac{q^{2}}{4}\right)^{1 / 2} C_{D}-\frac{q^{2} C_{D, P}}{3\left(4 M \omega-q^{2}\right)^{3 / 2}}+\left[\frac{q}{\sqrt{4 M \omega-q^{2}}}+4 \sinh ^{-1}\left(\frac{q}{\sqrt{4 M \omega-2 q^{2}}}\right)\right] \frac{C_{v}}{q} \\
& +\frac{2 q^{2}\left(7 q^{4}-40 q^{2} M \omega+60 M^{2} \omega^{2}\right)}{3\left(2 M \omega-q^{2}\right)^{2}\left(4 M \omega-q^{2}\right)^{5 / 2}} C_{v, P}+\frac{q^{4}-20 q^{2} M \omega+60 M^{2} \omega^{2}}{\left(2 M \omega-q^{2}\right)^{2}\left(4 M \omega-q^{2}\right)^{3 / 2}} C_{R} .
\end{aligned}
$$

For $\epsilon_{q}>\omega>\epsilon_{q} / 2, \quad I_{\mathrm{Ra}}(\mathbf{q}, \omega)$ is given by Eq. (28) with the factor $\sinh ^{-1}\left[q / \sqrt{4 M \omega-2 q^{2}}\right]$ replaced by $\cosh ^{-1}\left[q / \sqrt{-4 M \omega+2 q^{2}}\right] . I_{\mathrm{Ra}}(\mathbf{q}, \omega)=0$ when $\omega<\epsilon_{q} / 2$. In the limit $q \rightarrow 0, I_{\mathrm{Ra}}(\mathbf{0}, \omega)$ gives the radio-frequency response and involves only $C_{v}, C_{D}$, and $C_{R}$. The presence of $C_{D, P}$ and $C_{v, P}$ in Eq. (28) can also be understood from a Galilean covariance argument similar to the one given below Eq. (24).

\section{DISCUSSION}

The construction of the effective-field theory equation (1) for $d$-wave resonance suggests a general procedure for resonances of arbitrary higher partial waves. Consider a two-component Fermi gas with short-range interactions; the phase shift in the $\ell$ th scattering channel can be written as $k^{2 \ell+1} \cot \delta_{\ell}(k)=-\sum_{\alpha=0}^{\ell} k^{2 \alpha} / a_{\ell \alpha}+O\left(k^{2 \ell+2}\right)$ in the lowenergy limit. To reproduce the phase shift, we need only to generalize the dimer field term in Eq. (1) to

$$
\mathcal{L}_{d}=\sum_{m=-\ell}^{\ell} \sum_{\alpha=0}^{\ell} d_{\ell m}^{\dagger} \bar{z}_{\ell \alpha}\left(i \partial_{t}+\frac{\nabla^{2}}{4 M}\right)^{\alpha} d_{\ell m}
$$

and assume $L_{f d}$ to be of the form of Eq. (3) with the factor $\bar{g} \sqrt{4 \pi / V}$ replaced by $4 \pi / \sqrt{M V}$, which amounts to a rescaling of the dimer field $d_{\ell m}$. The relation between parameters $\left\{\bar{z}_{\ell \alpha}\right\}$ and the physical scattering parameters $\left\{a_{\ell \alpha}\right\}$ can be established similarly by matching the scattering $T$ matrix to that of $k^{2 \ell+1} \cot \delta_{\ell}(k)$. One finds

$$
\frac{1}{a_{\ell \alpha}}=\bar{z}_{\ell \alpha} M^{\alpha}+\frac{2}{\pi} \frac{\Lambda^{2(\ell-\alpha)+1}}{2(\ell-\alpha)+1}
$$

for $0 \leqslant \alpha \leqslant \ell$. For fixed $\left\{a_{\ell \alpha}\right\}$, the zero-range limit $\Lambda \rightarrow \infty$ is attainable only if $\bar{z}_{\ell \alpha}$ are all negative.

Experimental determination of the values of the contacts can be a challenge. In cold atomic gases, usually, only the leading scattering parameter, which is $D$ in the $d$-wave case, can be well controlled by the technique of Feshbach resonance; Eq. (17) can be practically used to determine $C_{D}$ only if the (free) energy of the system can be measured as a function of $D$ [30]. Fitting the tails of the momentum distribution or spectroscopic spectrums beyond the leading order is likely to suffer a low signal-to-noise ratio. However, extraction of the subleading order has been achieved in Fermi gases close to a $p$-wave resonance [4]. Measuring higher-order corrections to the observables would be more challenging.

\section{ACKNOWLEDGMENTS}

We thank H. Zhai, L.-F. Li, Z. Shi, and Y. Nishida for helpful discussions. This work is supported by the Tsinghua University Initiative Scientific Research Program, National Natural Science Foundation of China Grant No. 11474179. S.Z. is supported by the Hong Kong Research Grants Council (General Research Fund, HKU 17306414, and Collaborative Research Fund, HKUST3/CRF/13G) and the Croucher Innovation Awards.
[1] C. C. Tsuei and J. R. Kirtley, in Phase-Sensitive Tests of Pairing Symmetry in Cuprate Superconductors, edited by K. H. Bennemann and J. B. Ketterson (Springer, Berlin, 2008), Vol. 2.

[2] J. Werner, A. Griesmaier, S. Hensler, J. Stuhler, T. Pfau, A. Simoni, and E. Tiesinga, Phys. Rev. Lett. 94, 183201 (2005).

[3] Q. Beaufils, A. Crubellier, T. Zanon, B. Laburthe-Tolra, E. Maréchal, L. Vernac, and O. Gorceix, Phys. Rev. A 79, 032706 (2009).

[4] C. Luciuk, S. Trotzky, S. Smale, Z. Yu, S. Zhang, and J. H. Thywissen, Nat. Phys. 12, 599 (2016).

[5] S. Tan, Ann. Phys. (NY) 323, 2952 (2008); 323, 2971 (2008); 323, 2987 (2008).

[6] E. Braaten and L. Platter, Phys. Rev. Lett. 100, 205301 (2008).

[7] S. Zhang and A. J. Leggett, Phys. Rev. A 79, 023601 (2009).

[8] F. Werner, L. Tarruell, and Y. Castin, Eur. Phys. J. B 68, 401 (2009).
[9] E. Braaten, D. Kang, and L. Platter, Phys. Rev. Lett. 104, 223004 (2010).

[10] M. Valiente, N. T. Zinner, and K. Mølmer, Phys. Rev. A 84, 063626 (2011).

[11] M. Valiente, N. T. Zinner, and K. Mølmer, Phys. Rev. A 86, 043616 (2012).

[12] S. M. Yoshida and M. Ueda, Phys. Rev. Lett. 115, 135303 (2015).

[13] Z. Yu, J. H. Thywissen, and S. Zhang, Phys. Rev. Lett. 115, 135304 (2015).

[14] M. He, S. Zhang, H. M. Chan, and Q. Zhou, Phys. Rev. Lett. 116, 045301 (2016).

[15] X. Cui, Phys. Rev. A 94, 043636 (2016).

[16] D. Kaplan, Nucl. Phys. B 494, 471 (1997).

[17] S. J. J. M. F. Kokkelmans, J. N. Milstein, M. L. Chiofalo, R. Walser, and M. J. Holland, Phys. Rev. A 65, 053617 (2002).

[18] E. Braaten, P. Hagen, H.-W. Hammer, and L. Platter, Phys. Rev. A 86, 012711 (2012). 
[19] C. Langmack, M. Barth, W. Zwerger, and E. Braaten, Phys. Rev. Lett. 108, 060402 (2012).

[20] Y. Nishida and D. T. Son, Phys. Rev. Lett. 97, 050403 (2006).

[21] E. Braaten, M. Kusunoki, and D. Zhang, Ann. Phys. (NY) 323, 1770 (2008).

[22] K. G. Wilson, Phys. Rev. 179, 1499 (1969).

[23] E. Braaten, D. Kang, and L. Platter, Phys. Rev. A 78, 053606 (2008).

[24] D. T. Son and E. G. Thompson, Phys. Rev. A 81, 063634 (2010).

[25] E. Braaten, D. Kang, and L. Platter, Phys. Rev. Lett. 106, 153005 (2011).

[26] J. Hofmann, Phys. Rev. A 84, 043603 (2011).

[27] M. Barth and W. Zwerger, Ann. Phys. (NY) 326, 2544 (2011).
[28] W. D. Goldberger and Z. U. Khandker, Phys. Rev. A 85, 013624 (2012).

[29] M. E. Peskin and D. V. Schroeder, An Introduction to Quantum Field Theory (Westview, Boulder, 1995).

[30] J. T. Stewart, J. P. Gaebler, T. E. Drake, and D. S. Jin, Phys. Rev. Lett. 104, 235301 (2010).

[31] P. Makotyn, C. E. Klauss, D. L. Goldberger, E. A. Cornell, and D. S. Jin, Nat. Phys. 10, 116 (2014).

[32] S.-G. Peng, X.-J. Liu, and H. Hu, Phys. Rev. A 94, 063651 (2016).

[33] F. Qin, X. Cui, and W. Yi, Phys. Rev. A 94, 063616 (2016).

[34] J. P. Gaebler, J. T. Stewart, T. E. Drake, D. S. Jin, A. Perali, P. Pieri, and G. C. Strinati, Nat. Phys. 6, 569 (2010).

[35] M. Feld, B. Fröhlich, E. Vogt, M. Koschorreck, and M. Köhl, Nature (London) 480, 75 (2011). 